\title{
Methoden der direkten Blutammoniakbestimmung ohne Destillation
}

\author{
Von A. Englhardt, B. Haertwig, F. Frankin \\ Aus dem Zentrallaboratoriun der 2. Medizinischen Klinik.(Dircktor: Prof. Dr. K. Oberdissc) der Universilät Diisseldorf \\ und dem Laboratorium der Mediziniscben Klinik (Direktor: Prof. Dr. G. A. Martini) der Universitär Marburg/Lalın
}

(Eingegangen am 18. Dezember 1969 / 6. August 1970)

Folgende Methoden der direkten Ammoniakbestimmung im Vollblut wurden geprüft: kolorimetrische Bestimmung mit der PhenolHypochlotit-Reaktion in 2 Modifikationen und Bestimmung auf cnzymatischem Weg mit 2-Oxoglutarat, NADI-I und Glutamatdehydrogenase.

Fchlerquellen aller Blutammoniakbestimmungen finden sich vor allem in den Teilschritten von der Blutentnahme bis zum Reaktionsansatz.

Bei det Phenol-1-Hypochlorit-Reaktion rengieren Aminosäuren in verschicdenem Ausmaß. Eine quantitativ me(3bare Renktion wird nur von Glycin, Leucin, sowie sehr hohen Konzentrationen von Glutamin gegeben. İine Modifikation der Reaktion mit crhöhter Konzentration der Reagenzien ergab höhere Blutammoniakwerte aus densclben Blutproben als dic Modifikation nach MüLLLıR-BerssuNı1ıRT\% und KinLLER.

Positive Korrelationen fanden sich für die enzymatische und die Phenol---lypochloritmethode, sowic für die beiden Modifikationen der. letzteren.

Dic Wiederfindungsratc für dic Messung von zugesetztem $\mathrm{NH}_{3}-\mathrm{N}$ zu Vollblut war $98-100 \%$ für die kolorimetrische Methode. $Z$ wischen Extinktion bei $578 \mathrm{~nm}$ und $\mathrm{NH}_{3}-\mathrm{N}-$ Gehalt der Standardlösung bestand cinc streng lincare Bezichung.

Die Reproduzierbarkeit der beiden Methoden mit Phenol-Hypochlorit wurde durch Viclfachbestimmungen in der Serie und Doppelbestimmungen von Tag zu Tag geprüft. Der Variationskoeffizient für Bestimmungen in der Serie beträgt für die beiden Methoden 1,83 und 2,01\%, für dic Doppelbestimmungen 2,2 und 2,71\%.

Eigene Untersuchungen ergaben eine gute Übereinstimmung der Werte der Phenol-Hypochlorit-Methode aus eigenen Untersuchungen mit den Werten von MülLer-Buissenhirtrz und Keller im Normbercich.

\section{Methods for the direct determination of blood ammonia without distillation}

In the present serics of investigations the following methods were used for the direct detcrmination of ammonia in whole blood: colorimetric detcrmination by 2 modifications of the phenol-hypochloritc renction and the cnzymic determination with 2-Oxoglutarate, NADH - and glutamic acid dehydrogenasc.

The chicf sources of error in all detcrminations of blood ammonin are located in the stages from the sampling of the blood to the analytical reaction mixture.

$\Lambda$ mino acids react to varying extents in the phenol-hypochlorite renction. Quantitatively measurable renctions are only given by glycinc, leucine and by very high concentrations of glutamine. A modification of the reaction with increased concentrations of reagents gave higher blood ammonia values than the modification of MüLLER-BEISSENHIRTz and KHILLRR, using the same blood samples. There were positive correlations between the enzymic and the phenol-hypochlorite methods, and between the two modifications of the latter.

The colorimetric method showed a $89-100 \%$ recovery for $\mathrm{NH}_{3}-\mathrm{N}$ added to whole blood. There was a strict linear relationship between the extinction at $578 \mathrm{~nm}$ and the $\mathrm{NH}_{3}-\mathrm{N}$ concentration of the standard solution.

The reproducibility of the two phenol-hypochlorite methods was tested by multiple clecerminations in scrics and by double measurements from day to day. $\mathrm{V} \%$ in the series for the two methods was 1.83 and $2.01 \%$, and for the double determinations $2.2 \%$ and $2.71 \%$. Within the normal range, single determinations showed good agreement between values obtained by the phenolhypochloritc method and the valucs of MÜLLZR-BeISSENHIR'TZ and KRLLER.

Ammoniakbestimmungen zur Diagnostik der Leberinsuffizienz wurden wegen der schwierigen und aufwendigen Methoden bisher in Routinelaboratorien kaum durchgeführt. Diese Methoden beruhten auf dem Prinzip, das $\mathrm{NH}_{4}$-Ion durch Alkalisieren in Ammoniak überzuführen $(1,13)$. In letzter Zeit sind Verfahren beschrieben worden, die eine direkte Bestimmung des Ammoniak in biologischem Material ohne Destillation crlauben. Eine Methode zur Bestimmung in Trichloressigsäurcextrakten wurde von MülLIER-BEIsszNHIRTZ und KeLler (2) entwickelt. Hicr wird Ammoniak mit Hilfe der BERTHELotschen Reaktion (3) nachgewiesen. Eine Modifikation wurde von Breuer (4) beschricben. Eine weitere Möglichkeit ist dic enzymatische Bestimmung nach Enteiweißung im neutralen Milicu unter
Zusatz von 2-Oxoglutarat und Glutamatdehydrogenase im optischen Test $(5,6)$.

Dic Bcwertung der Methoden ist unterschicdlich. Schmrdt und Scrwarz (6) geben an, daß die Hypochlorit-Phenolatmethode zwar cinfacher in der Durchführung ist, aber. Fchlercquellen hat, auf dic im einzclnen nicht eingegangen wird.

In letzter Zeit wurde die dingnostische Bedeutung der Blutammoniakbestimmung wicder diskutiert, da dic Entwicklung der Methoden der dirckten Ammoniakbestimmung ihte Durchführung auch im Routinelaboratorium crmöglicht. In eigenen Untersuchungen wurden Ammoniakbestimmungsmethoden angewendet: und geprüft, wobei Erfahrungen anderer Autoren berücksichtigt wurden $(2,7,8,9)$. 


\section{Methodik}

Phenol-Hypochloritreaktion

Reagenzien

Phenol p. a. Merck; Dinatriumpentacyanonitrosylferrat Merck; $\mathrm{Na}_{2} \mathrm{HPO}_{4} \cdot 12 \mathrm{H}_{2} \mathrm{O}$, Merck; $\mathrm{NaOH}$ in rotulis Merck; Natriumhypochlorit-Lösung (Merck) mit $13 \%$ aktivem Chlor. Die Prüfung des Gehalts an aktivem Chlor wurde nach der Methode

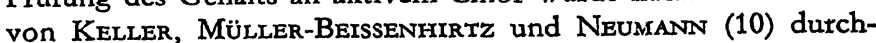
geführt. $\left(\mathrm{NH}_{4}\right)_{2} \mathrm{SO}_{4}$ p. A. zur Herstellung der Eichlösungen. Die Herstellung der Lösungen für die Methode von MüLLERBeIsSENHIRTZ und KeLLer erfolgte nach Angaben der Autoren (2).

Lösungen für die modifizierte Methode:

Lösung A: $20 \mathrm{~g}$ Phenol und $100 \mathrm{mg}$ Dinatriumpentacyanonittosylferrat werden getrennt gelöst, gemischt und mit bidest. Wasser auf $1 l$ aufgefüllt.

Lösung B: $180 \mathrm{~g} \mathrm{Na}_{2} \mathrm{HPO}_{4} \cdot 12 \mathrm{H}_{2} \mathrm{O}$ und $20 \mathrm{~g} \mathrm{NaOH}$ werden getrennt gelöst und mit frischem dest. Wasser auf etwa $800 \mathrm{ml}$ aufgefüllt. Nach Abkühlen im Eisbad Zusatz von $20 \mathrm{~m} /$ Natriumhypochloritlösung mit $4,5 \%$ Gehalt an aktivem Chlor (durch Verdünnung mit bidest. Wasser aus der 13 proz. Lösung herstellen). pH dieser Lösung 12,6-12,9. Anschließend wird mit frischem bidest. Wasser ad 1 l aufgefüllt.

Eichlösung: 4,72 $\mathrm{g} \quad\left(\mathrm{NH}_{4}\right)_{2} \mathrm{SO}_{4}$ werden mit frischem bidest. Wasser gelöst und auf $1 \mathrm{l}$ aufgefüllt. Diese Lösung enthält $100 \mathrm{mg} /$ $100 \mathrm{~m} l \mathrm{NH}_{3}-\mathrm{N}$. Aus dieser Stammlösung werden Verdünnungen für Eichkurven und Standardlösungen hergestellt.

\section{Durcbfübrung der Bestimmung (2)}

Etwa $1 \mathrm{ml}$ Blut wurde in einer $1 \mathrm{~m} /$-Mikroliterspritze abgezogen und sofort $0,5 \mathrm{~m} l$ in ein $\mathrm{Gefä} B \mathrm{mit} 0,5 \mathrm{ml}$ eisgekühlter 10 proz. $(w / v)$ Trichloressigsäure bzw. Trichloressigsäure-NaOH-Lösung (10 g Trichloressigsäure $+1,3 \mathrm{~g} \mathrm{NaOH}$ ad $100 \mathrm{ml}$ bidest. Wasser) gegeben. Es wurden jeweils 2-3 Ansätze in dieser Weise durchgeführt. Die Gefäße (Plastikgefäße zum Mikrolitersystem Eppendorf) wurden sofort gut geschüttelt und der Niederschlag in der Zentrifuge zum Mikrolitersystem abzentrifugiert. Aus jedem Gefäß wurden $0,5 \mathrm{ml}$ des klaren Überstands mit $2 \mathrm{ml}$ Phenolreagenz und $2 \mathrm{~m} l$ Hypochloritreagen $z$ gemischt und $30 \mathrm{Min}$. bei $37^{\circ}$ inkubiert. Im Anschluß daran wirden die Extinktionen am Photometer Eppendorf abgelesen (d=1 cm, $\lambda=578 \mathrm{~nm})$. Für die Modifikation der Methode mit Hypochlorit wurden $0,5 \mathrm{~m} /$ des klaren Überstands mit $6 \mathrm{~m} l$ Lösung A (Phenollösung) und $6 \mathrm{~m} l$ Lösung B (Hypochloritlösung) gemischt und wie oben inkubiert. Die Ablesung erfolgte wie oben bei $578 \mathrm{~nm}$, aber Schichtdicke $2 \mathrm{~cm}$.

Bei jeder Messung wird in beiden Methoden Leerwert und ein Standardwert $(200 \mu \mathrm{gN} / 100 \mathrm{~m} l)$ mitgeführt.

Berechnung:

$$
\frac{\text { Extinktion Probe }}{\text { Extinktion Standard }} \cdot 200=\mu \mathrm{g} \mathrm{NH}_{3}-\mathrm{N} / 100 \mathrm{~m} / \text { Blut }
$$

Enzymatische Bestimmung

Die Bestimmung auf enzymatischem Weg wurde nach der von SCHMidT und SCHwarz (6) angegebenen Methode durchgeführt, die in einigen Punkten modifiziert wurde (s. u.).

Die kinetische Messung wurde stets unmittelbar an die Enteiweißung angeschlossen. Es hat sich nämlich gezeigt, daß die Ammoniakneubildung in den sauren Extrakten in den einzelnen Blutproben in verschiedenem Umfang stattfindet. SCHMIDT und SCHWARz haben empfohlen, in Abhängigkeit von der Zeit zwischen Blutentnahme und Bestimmung mit Hilfe eines Korrekturfaktors auf die Ammoniakkonzentration zum Zeitpunkt $t_{0} z u$ schließen. In eigenen Untersuchungen gelang es aber nicht, einen für alle Blutproben gültigen Korrekturfaktor zu finden, da die Ammoniakbildungstate $z u$ unterschiedlich war.

Eine Schwierigkeit der Methode war, daß die Reaktion nicht immer, wie angegeben, nach $40 \mathrm{Min}$. beendet war, sondern in einen kontinuierlichen „Schleich" überging. Der eigentliche Endwert mußte durch Extrapolation gewonnen werden. Es war daher erforderlich, die Kinetik über einen längeren Zeitraum zu verfolgen, was mit größerem Zeitaufwand verbunden war. Auch war die angegebene Enzymmenge nicht immer ausreichend, um die Reaktion in der angegebenen Zeit zum vollständigen Ablauf zu bringen.

\section{Ergebnisse}

\section{Fehlerquellen der Methoden}

Die größten Fehlermöglichkeiten der Ammoniakbestimmung liegen in den Teilschritten von der Blutentnahme bis zur eigentlichen Messung. Bereits ConwaY und Mitarbeiter (1, 12-14) beschrieben eine Ammoniakneubildung in Blutproben in vitro aus verschiedenen Quellen, vor allem aus Adenosin, ATP und AMP. Auch der Glutamin-N-Spiegel des Blutes liegt fast 200 fach höher als der des $\mathrm{NH}_{3}-\mathrm{N}(16,17)$.

Es wurde daher immer wieder versucht, durch Extrapolieren den in vivo-Ammoniakspiegel des Blutes zu ermitteln. Conway und Cooke (13) ermittelten auf diese Weise, daß das strömende Blut Gesunder kein Ammoniak enthält. Dagegen sprechen die von einer Reihe von Autoren ermittelten $\mathrm{NH}_{3}-\mathrm{N}$-Bildungstaten, die $z$ wischen 5 und $35 \mu \mathrm{g} / \mathrm{Std} \cdot 100 \mathrm{~m} l$ Blut liegen. Eigene Ergebnisse stimmen mit diesen Werten überein (Tab. 1). Das bedeutet, daß unter Annahme eines

Tab. 1

$\mathrm{NH}_{3}-\mathrm{N}$-Bildungsrate in $\mu \mathrm{g} / 100 \mathrm{ml}$ Blut - Std. bei Aufbewahrung der Blutproben, bzw, der enteiweißten Extrakte

Werte nach Angaben verschiedener Autoren und eigene Ergebnisse

\begin{tabular}{|c|c|c|c|c|}
\hline $\begin{array}{c}\text { Unter- } \\
\text { suchungs- } \\
\text { material }\end{array}$ & $\begin{array}{c}\text { Temperatur } \\
{\left[{ }^{\circ} \mathrm{C}\right]}\end{array}$ & $\mathrm{n}$ & $\begin{array}{l}\mathrm{NH}_{3}-\mathrm{N}-\mathrm{Bil}- \\
\text { dungsrate } \\
\mu \mathrm{g} / 100 \mathrm{ml} \\
\text { Std. }\end{array}$ & Autoren \\
\hline
\end{tabular}

a) Aufbewahrung des Nativbluts

\begin{tabular}{|c|c|c|c|c|}
\hline Vollblut & $20-22$ & & $5,10-34,8$ & $\begin{array}{l}\text { MÜLLER-BEI- } \\
\text { SSENHIRTZ u. } \\
\text { KELLER (2) }\end{array}$ \\
\hline Vollblut & 0 & & 60,0 & $\begin{array}{l}\text { CONWAY u. } \\
\text { COOKE (1I) }\end{array}$ \\
\hline Vollblut & 0 & & 42,0 & KIRK (23) \\
\hline Vollblut & 0 & & 18,0 & $\begin{array}{l}\text { SELIGSON } u \\
\text { HIRAHARA }(21)\end{array}$ \\
\hline Vollblut & 0 & & 10,1 & FORMAN (20) \\
\hline Vollblut & 0 & & 5,5 & $\begin{array}{l}\text { MILLER u. } \\
\text { RICE (22) }\end{array}$ \\
\hline Vollblut & 0 & 12 & 15,6 & $\begin{array}{l}\text { eigene Unter- } \\
\text { suchungen }\end{array}$ \\
\hline
\end{tabular}

b) Aufbewahrung der tiefgefrorenen Probe nach Einfrieren in flüssiger Luft

Vollblut

20,11

eigene Unter-

c) Aufbewahrung der enteiweißten Extrakte $\begin{array}{lllll}\text { Trichloressigsäure- } & 0 & 8 & 19,0-88,0 & \begin{array}{l}\text { eigene Unter- } \\ \text { Extrakt }\end{array}\end{array}$

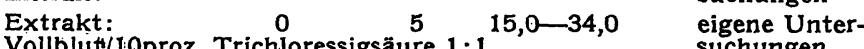
Vollblut/10proz. Trichloressigsäure $1: 1^{15,0-34,0}$ suchungen Extrakt: $\quad 0 \quad 5 \quad 4,0-30,0$ eigene UnterVollblut/10proz. Trichloressigsäure $+1,3 \% \mathrm{NaOH} 1: 1$. suchungen Vollblut/10proz. Trichloressigsäure 1:1 dazu 6,65proz. $\mathrm{K}_{3} \mathrm{PO}, 3,0 \mathrm{ml}$ zu 5,0 ml Extrakt

linearen Anstiegs der Blutammoniakwerte in vitro ein Spiegel von $60 \mu \mathrm{g} / 100 \mathrm{~m} l$ frühestens $2 \mathrm{Stdn}$. nach der Entnahme erreicht werden kann. Wir haben durch fortlaufende Ammoniakbestimmungen aus einer Blutprobe den Verlauf der Ammoniakneubildung verfolgt und die Kurve gegen $t_{0}$ extrapoliert (Abb. 1). Es ergab sich ein in vivo-Wert von im Mittel $68 \mu \mathrm{g} / 100 \mathrm{ml}$ im 


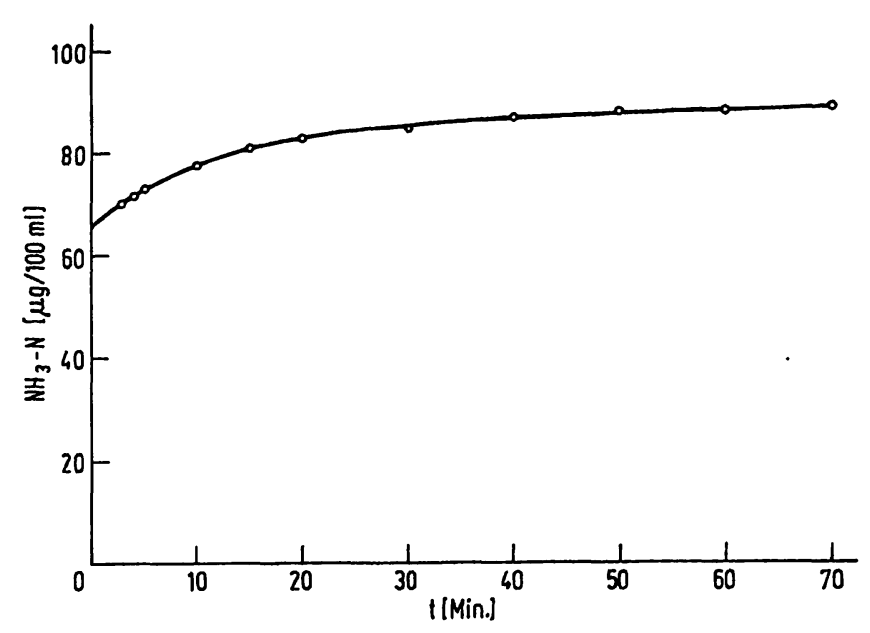

Abb. 1

$\mathrm{NH}_{2}-\mathrm{N}$-Bildungsrate im Vollblut

Blutentnahmen aus einer Blutprobe in Abständen bis zu 70 Min Aufbewahrung der Probe während des Versuchs bei $+4^{\circ}$. Enteiweißung $(0,5 \mathrm{ml}$ Blut $+0,5 \mathrm{ml}$ 10proz. Trichloressigsäure eisgekühlt) sofort nach Entnahme, Zentrifugieren 2 Min., Zentrifuge des Mikrolitersystems Eppendorf. Anschließend Reaktionsansatz (siehe Methodik). Die erhaltene Kurve wurde nach $t_{0}$ extrapoliert

Blut. Derselbe Wert wurde gewonnen, wenn eine Blutprobe $(0,5 \mathrm{~m} l)$ sofort nach der Entnahme in flüssige Luft gebracht wurde und anschließend die Enteiweißung durch Zusatz von $0,5 \mathrm{ml} 10$ proz. Trichloressigsäure, wie in der Methodik beschrieben, durchgeführt wurde.

\section{Enteiveißung}

$\mathrm{Da}$ sowobl im sauren wie im alkalischen Milieu eine nicht enzymatische Desaminierung stattfindet, schreitet die Ammoniakneubildung auch nach Enteiweißung weiter fort. Im sauren Trichloressigsäuremilieu ist die $\mathrm{NH}_{3}-\mathrm{N}$-Bildungsrate sogar höher als im Vollblut (Tab. 1). KeLLeR und Mitarbeiter (10) verwenden daher zur Enteiweißung eine Trichloressigsäurelösung, die zur Hälfte mit $\mathrm{NaOH}$ neutralisiert ist. In eigenen Untersuchungen wurde der Umfang der $\mathrm{NH}_{3}-\mathrm{N}-\mathrm{Neu}-$ bildung nach Enteiweißung in verschiedenen Extrakten geprüft. Im Trichloressigsäureextrakt war sie von Probe zu Probe sehr unterschiedlich (10-78\%/ Std., im Mittel 38\%/Std.). Durch Zusatz von $\mathrm{NaOH}$ konnte die Zuwachsrate auf 16-28\%/Std., im Mittel $22 \% /$ Std. gesenkt werden. Die besten Ergebnisse erhielten wir, wenn die Fällung zunächst mit Trichloressigsäure vorgenommen wurde und unmittelbar danach die Neutralisation mit $\mathrm{K}_{3} \mathrm{PO}_{4}$ durchgeführt wurde (Zuwachsrate 3-25\%/Std., im Mittel 15\%/ Std. (Abb. 2). Der ursprüngliche $\mathrm{NH}_{3}-\mathrm{N}-\mathrm{Gehalt}$ wurde dabei gleich $100 \%$ gesetzt.

\section{Phenol-Hypocbloritreaktion}

Die meisten Verfahren der kolorimetrischen Ammoniakbestimmung im Blut bedienen sich der Phenol-Hypochlorit-Reaktion (BERTHELOTsche Reaktion). Die Reaktion ist, wie bereits die ersten Untersucher $(17,3)$ nachweisen konnten, keineswegs spezifisch für Ammoniak, da Aminosäuren, vor allem Glycin, ebenfalls mit Farbentwicklung reagieren. Nach LANGHELd (18)

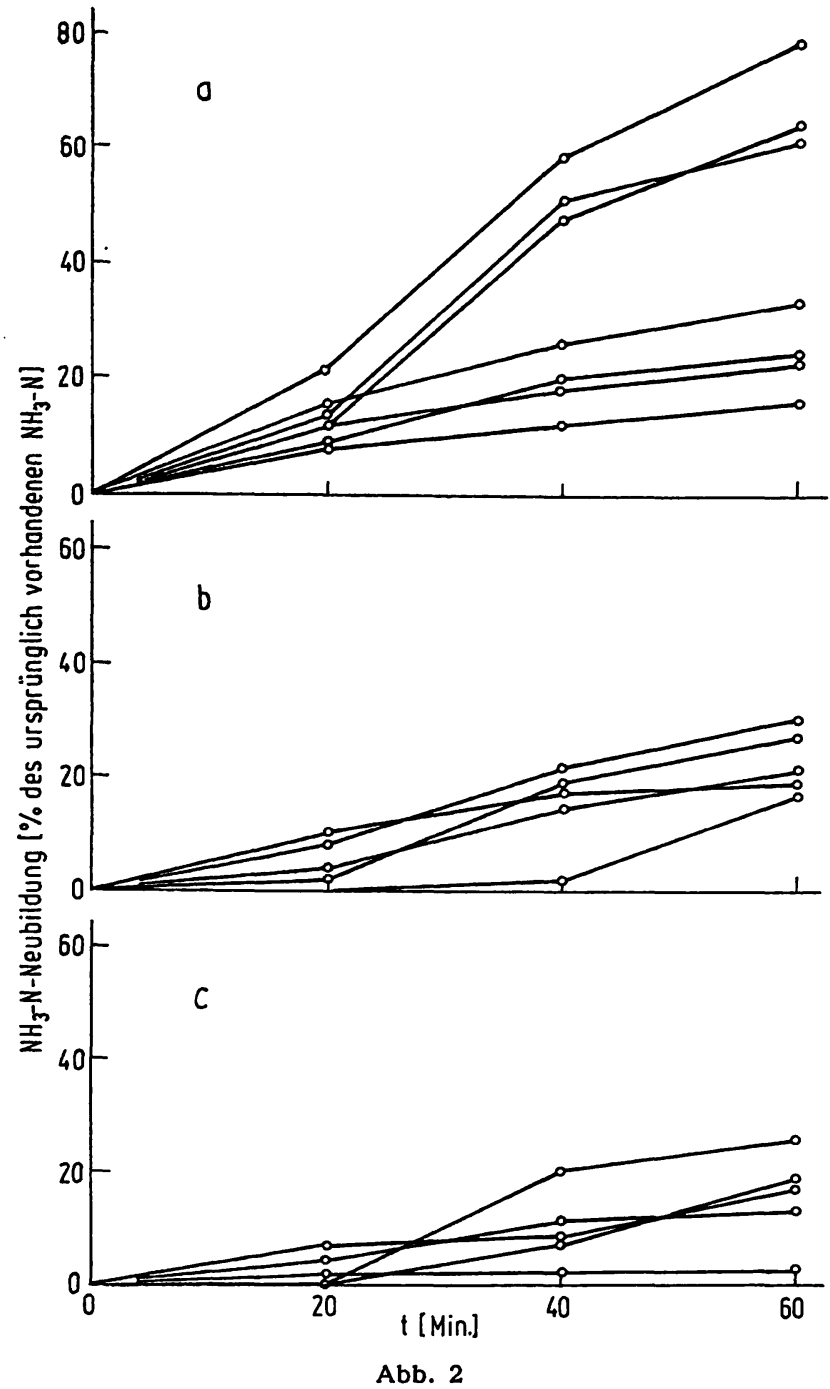

$\mathrm{NH}_{3}-\mathrm{N}$-Bildung in eiweißfreien Extrakten aus menschlichem Vollblut a) Blut/10proz. Trichloressigsäure 1:1. b) Blut/10proz. Trichloressigsäure $+1,3 \% \mathrm{NaOH}$. c) Blut/10proz. Trichloressigsäure, neutralisiert mit 6,65 proz. $\mathrm{K}_{3} \mathrm{PO}_{4} \cdot 3 \mathrm{H}_{2} \mathrm{O} ; 5+3(\mathrm{v} / \mathrm{v})$

Tab. 2 a

Phenol-Hypochlorit-Reaktion mit Vollblut mit und ohne Zusatz Stickstoffhaltiger Substanzen

Die Substanzen wurden dem Vollblut sofort nach der Entnahme zugesetzt. Endkonzentration im Blut siehe Tabelle. Unmittelbar im Anschluß daran Enteiweißung mit 10proz. Trichloressigsäure und Durchführung der Phenol-Hypochlorit-Reaktion nach MüLLERBEISSENHIRTZ und KELLER
$\mathrm{NH}_{\mathbf{3}}-\mathrm{N}$-Gehalt der Blutprobe ohne Zusatz = 100proz.

\begin{tabular}{lcc}
\hline Zusatz & $\begin{array}{c}\text { Endkonzentration } \\
\text { der zusammenge- } \\
\text { setzten Substanzen } \\
\text { im Blut (mg/100 ml) }\end{array}$ & $\begin{array}{c}\text { Anstieg bzw. Abfall } \\
\text { des N } \text { N }_{3} \text {-N } \\
\text { Kontrolle = 100\% }\end{array}$ \\
\hline Ohne Zusatz & - & 100,0 \\
Harnstoff & 30,0 & 102,4 \\
Harnstoff & 60,0 & 105,2 \\
Harnsäure & 2,0 & 101,3 \\
Harnsäure & 20,0 & 105,3 \\
Glycin & 2,0 & 105,0 \\
Glycin & 4,0 & 116,4 \\
Alanin & 4,0 & 98,3 \\
Alanin & 40,0 & 97,4 \\
Leucin & 2,0 & 120,5 \\
Leucin & 4,0 & 128,8 \\
Glutamin & 6,0 & 106,8 \\
Glutamin & 60,0 & 117,8 \\
Kreatinin & 10,0 & 105,0 \\
ATP & 40,0 & 98,2 \\
NADH & 2,0 & 103,5 \\
0,9proz. NaCl & - & 98,2 \\
\hline
\end{tabular}

werden $\alpha$-Aminosäuren durch Hypochlorit zu Aldehyden abgebaut, wobei der Stickstoff der Aminogruppe als Ammoniak in Freiheit gesetzt wird. Bei Überschuß von Hypochlorit setzt sich Ammoniak weiter zu 
Tab. $2 b$

Phenol-Hypochlorit-Reaktion bei verschiedener Inkubationsdauer und Inkubationstemperatur

Methode nach Müller-Beissenhirtz und KELler. Messung von Methode nach
Vollblutproben und Ammoniumsulfat-Standardiösungen

\begin{tabular}{|c|c|c|c|c|}
\hline Probe & $\begin{array}{c}\text { Inkubations- } \\
\text { dauer } \\
\text { (Min.) }\end{array}$ & $\begin{array}{c}\text { Inkubations- } \\
\text { temperatur } \\
{\left[{ }^{\circ} \mathrm{C}\right]}\end{array}$ & $E_{B 78}$ & $\begin{array}{l}\text { Farbent- } \\
\text { wicklung }\end{array}$ \\
\hline Vollblut & $\begin{array}{r}30 \\
15 \\
5\end{array}$ & $\begin{array}{l}37 \\
60 \\
95\end{array}$ & $\begin{array}{l}0,114 \\
0,087 \\
0,069\end{array}$ & $\begin{array}{l}\text { blaugrün } \\
\text { grün } \\
\text { gelb }\end{array}$ \\
\hline Vollblut & $\begin{array}{r}30 \\
15 \\
5\end{array}$ & $\begin{array}{l}37 \\
60 \\
95\end{array}$ & $\begin{array}{l}0,124 \\
0,089 \\
0,061\end{array}$ & $\begin{array}{l}\text { blaugrün } \\
\text { grün } \\
\text { gelb }\end{array}$ \\
\hline Vollblut & $\begin{array}{r}30 \\
15 \\
5\end{array}$ & $\begin{array}{l}37 \\
60 \\
95\end{array}$ & $\begin{array}{l}0,200 \\
0,156 \\
0,154\end{array}$ & $\begin{array}{l}\text { blaugrün } \\
\text { grün } \\
\text { grün }\end{array}$ \\
\hline $\begin{array}{l}\text { Standard } \\
200 \mu \mathrm{g} ! 100 \mathrm{ml}\end{array}$ & $\begin{array}{r}30 \\
15 \\
5\end{array}$ & $\begin{array}{l}37 \\
60 \\
95\end{array}$ & $\begin{array}{l}0,220 \\
0,242 \\
0,250\end{array}$ & $\begin{array}{l}\text { blaugrün } \\
\text { blaugrün } \\
\text { blaugrün }\end{array}$ \\
\hline $\begin{array}{l}\text { Standard } \\
200 \mu \mathrm{g} / 100 \mathrm{ml}\end{array}$ & $\begin{array}{r}30 \\
15 \\
5\end{array}$ & $\begin{array}{l}37 \\
60 \\
95\end{array}$ & $\begin{array}{l}0,200 \\
0,220 \\
0,248\end{array}$ & $\begin{array}{l}\text { blaugrün } \\
\text { blaugrün } \\
\text { blaugrün }\end{array}$ \\
\hline
\end{tabular}

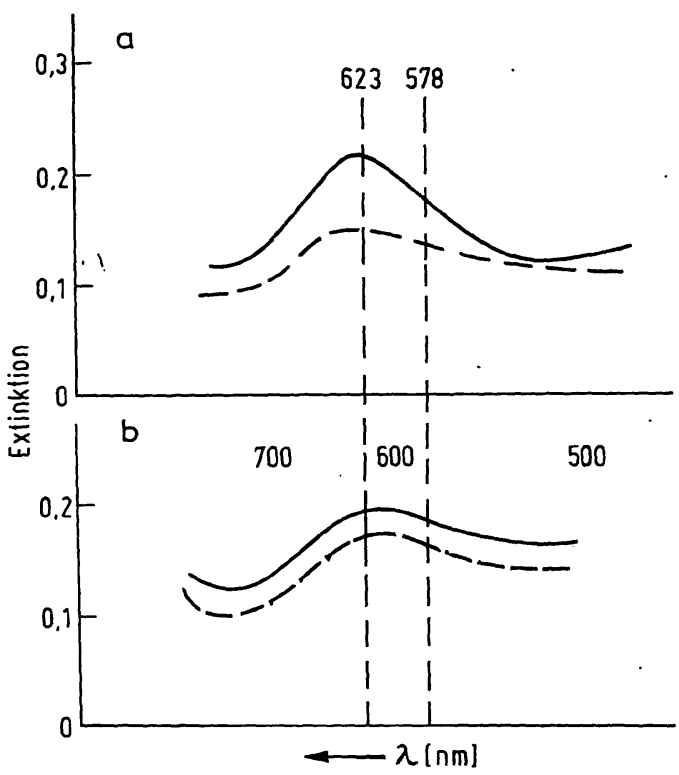

Abb. $3 a$

Änderung der Absorption durch Variation des Testansatzes Phenol-Hypochlorit-Reaktion Standard $100 \mu \mathrm{g} / 100 \mathrm{ml}--$ Blut. a) Methode nach MüLlER-BEISSENHIRTZ und KELLER (2) b) Modifikation der Methode (siehe Methodik)

Chloramin um, wobei freier Stickstoff entsteht (19). Die Farbreaktion wird also durch die Hypochloritkonzentration im Ansatz stark beeinflußt. Aber auch die Inkubationsdauer ist von Bedeutung. Nach FORMAN (20) stören Aminosäuren nur bei langer Inkubationszeit. In eigenen Untersuchungen fanden wir, daß außer Aminosäuren auch Nucleotide die Reaktion beeinflussen, daß aber eine wesentliche Reaktion nur durch Glycin, Leucin und hohe Konzentrationen von Glutamin hervorgerufen wird (Tab. 2a). In noch höheren Konzentrationen wird die Farbentwicklung der BERTHELOTschen Reaktion durch Aminosäuren gehemmt (7, 10). Eine Verkürzung der Inkubationsdauer bei gleichzeitiger Erhöhung der Reaktionstemperatur brachte keine günstigeren Ergebnisse. Die Farbentwicklung des Standards wurde nicht beeinflußt, die der Probe aber stark gestört durch Entstehung gelblicher Farbtöne, wahrscheinlich durch Mitreaktion weiterer unbekannter Substanzen (Tab. 2b).

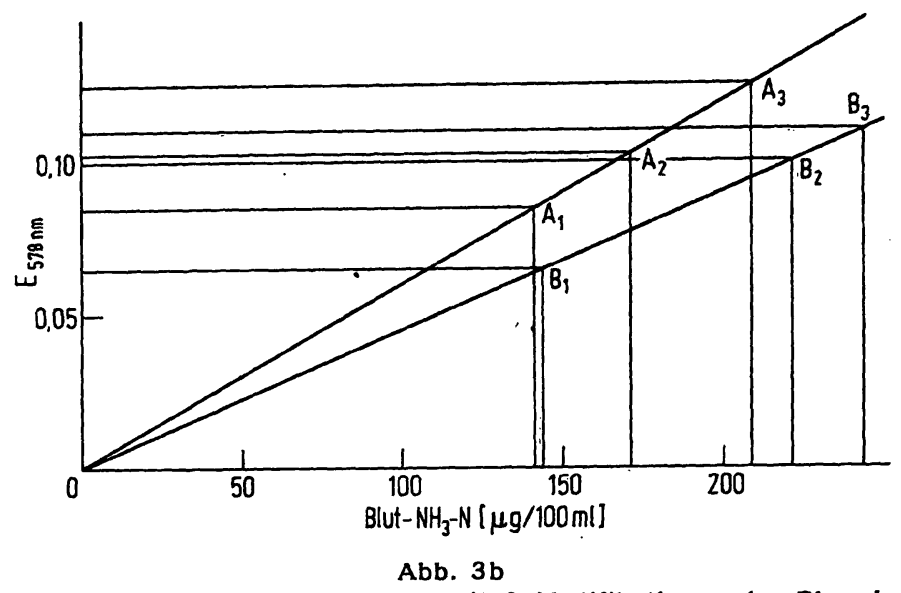

$\mathrm{NH}_{3}-\mathrm{N}$-Bestimmung im Vollblut mit 2 Modifikationen der PhenolHypochlorit-Reaktion

Die graphische Ermittlung des Blut-NH der mit den beiden Modifikationen bestimmt wurde, erfolgte durch direkte Ablesung aus den zugehörigen Eichkurven

Ansatz A: Reagenzien:
Phenol-Reagenz: $\quad 10 \mathrm{~g}$ Phenol-50 mg Dinatriumpentacyanonitrosylferrat auf 1 i

Hypochlorit-Reagenz: $90 \mathrm{~g} \mathrm{Na}_{2} \mathrm{HPO}_{4} \cdot 12 \mathrm{H}_{2} \mathrm{O}+10 \mathrm{~g}$

$\mathrm{NaOH}+3,8 \mathrm{ml} 13$ proz. Hypochloritlösung auf $1 \mathrm{l}$

Phenol-Reagenz: $20 \mathrm{~g}$ Phenol $+100 \mathrm{mg}$ Dinatriumpentacyanonitrosylferrat auf 1 l

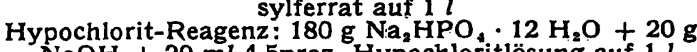

$\mathrm{NaOH}+20 \mathrm{ml} 4,5$ proz. Hypochloritlösung auf $1 \mathrm{l}$ Für $A$ und $B$ :

$0,5 \mathrm{ml}$ Trichloressigsäure-Blut-Gemisch 1:1, 2,0 ml Phenol-Reagenz, $2,0 \mathrm{ml}$ Hypochlorit-Reagenz

$\begin{array}{ll}\mathrm{A}_{1} 141 \mu \mathrm{g} / 100 \mathrm{ml} & \text { Blut-NH} \mathrm{H}_{3}-\mathrm{N}: \\ \mathrm{A}_{2} & \mathrm{~B}_{1} 145 \mu \mathrm{g} / 100 \mathrm{ml}\end{array}$

Auch durch Änderungen der Konzentrationen im Reaktionsansatz wurden Standard und Probe nicht in gleicher Weise beeinflußt. Erhöht man die Hypochloritkonzentration, so nimmt die Farbintensität des Standards ab (eigene Versuche). Möglicherweise wird in der Probe die Freisetzung des Stickstoffs aus Ammoniak durch andere Faktoren gehemmt. Erhöht man auch die Konzentration an Phenol und Dinatriumpentacyanonitrosylferrat, erhält man höhere Ammoniakwerte im Blut, da die Farbentwicklung des Standards stärker absinkt als die der Probe (Abb. 3a und b).

\section{Zuverlässigkeitskontrolle}

Die Prüfung der Richtigkeit der Ammoniakwerte ist schwierig, da wegen der Variabilität des Standards eine Eichung über einen Extinktionskoeffizienten nicht möglich ist. Daher wurden die Werte der kolorimetrischen Methode mit den auf enzymatischem Weg gewonnenen Werten verglichen. Es ergab sich eine Korrelation der beiden Methoden $(r=0,74)$, jedoch keine absolute Ubereinstimmung der Werte (Abb. 4). Ein Vergleich der beiden Modifikationen der PhenolHypochlorit-Reaktion zeigt ebenfalls bei ausreichender Korrelation Abweichungen vor allem im oberen Bereich, wobei die Modifikation der Methode mit höheren Konzentrationen an Phenol und Hypochlorit höhere Werte ergab.

Die Reproduzierbarkeit der Methoden wurde durch Vielfachbestimmungen in der Serie und durch Doppelbestimmungen von Tag zu Tag geprüft. Vielfachbestimmungen aus Extrakten verschiedener Blutproben 


\section{Wie lange dauert bei Ihnen eine wahre Glukose-Bestimmung?}

\section{Wir benötigen nur 10 Sekunden ...}
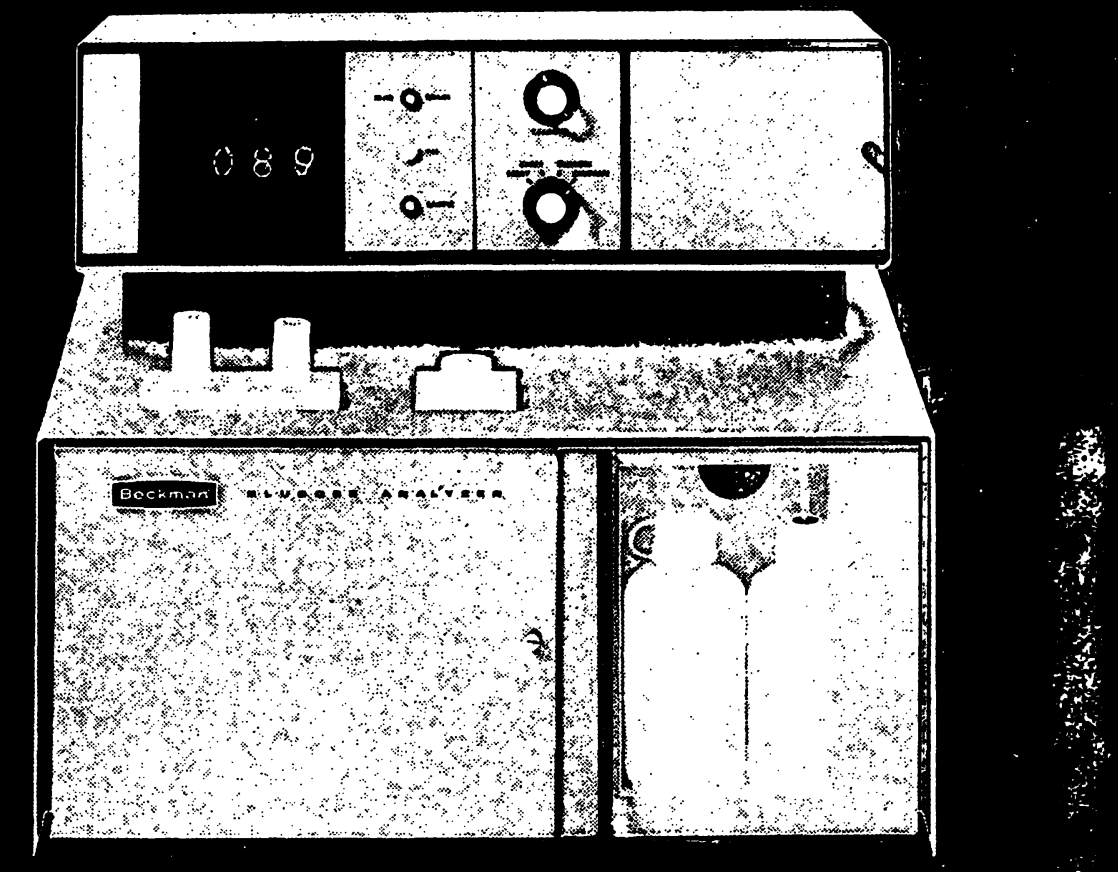

... mit dem neuen Beckman-Glukose-Analysator Modell ERA-2001 durch Anwendung einer völlig neuen Meßtechnik.

Unser Gerät ermittelt die wahre Glukose durch polarographische Messung der Abnahmegeschwindigkeit des Saụerștoffs während der enzymatischen Oxydation.

Weitere überzeugende Argumente:

( Mikroliter-Proben

$10 \mu \mathrm{l}$ Serum oder Plasma bzw. $50 \mu \mathrm{l}$ Urin

O Ohne Enteiweißung oder Probenverdünnung
Digitale Anzeige der Meßwerte in $\mathrm{mg} \%$ Glukose

(2) Meßgenauigkeit $\pm 2 \%$ Linearität der Anzeige bis $400 \mathrm{mg} \%$

Außerordentlich günstiger Preis

\section{Beckman}

BECKMAN INSTRUMENTS GMBH 8 München 45, Frankfurter Ring 115, Tel. 38871 , Telex 05-23823

Technische Büros: Berlln, Tel. 31210 35; Hamburg. Tel. 5195 54; Hannover, Tel. 6639 92; Düsseldorf, Tel. 6844 93; Frankfurt, Tel. (06103) 1003 ; Stuttgart, Tel. 71 18 37; München, Tel. 885035

Internationale Niederlassungen: Fullerton/USA, Genf, Paris,

Glenrothes/Schottland, Toklo, Kapstadt, Wien, Amsterdam, Stockholm 


\section{Automation Whres Laboratoriums!}

\author{
Eine Notwendigkeit!
}

\section{Begegnen Sie dem \\ Arbeitskräftemangel und wählen Sie MECOLAB M}

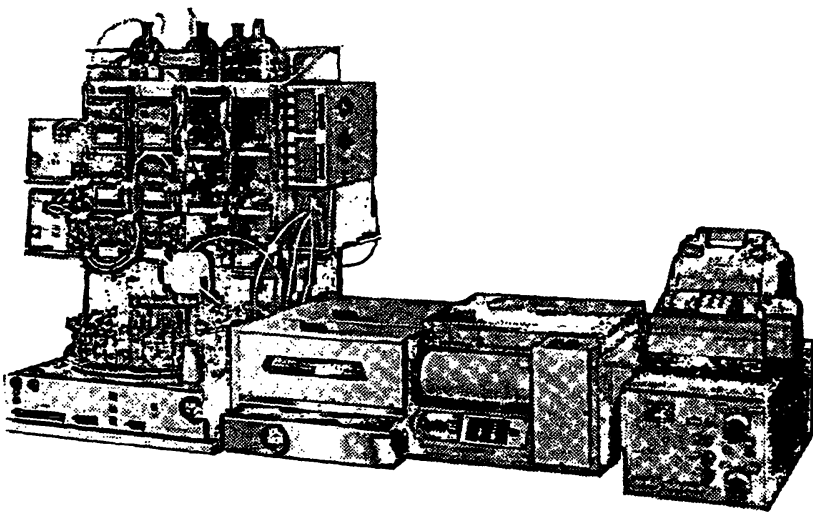

eines unserer Geräte zur Automation Ihres Laboratoriums.

Dieses Gerät gestattet:

- Automatische Durchführung mikrobiologischer Analysen ( $z$. B. Bestimmung von Folsäure, Vitamin $B_{6}$ und $B_{12}$ )

- Analyse von Probenchargen bis zu $\mathbf{4 0}$ Proben

- Mischung von Gesamtblut vor der Probenahme

- Erneute Suspendierung biologischer Substanzen vor der Photometrierung

- Möglichkeiten der zweimaligen oder dreimaligen Messung von Proben

- Analysengeschwindigkeit von 3 Proben pro Minute

- Methodenwechsel innerhalb von Minuten

Durch MECOLAB $M$ werden unsere bewährten automatischen Analysensysteme MECOLAB $C$ für klinischchemische Routineanalysen und MECOLAB E für Enzymkinetik um ein weiteres System bereichert.

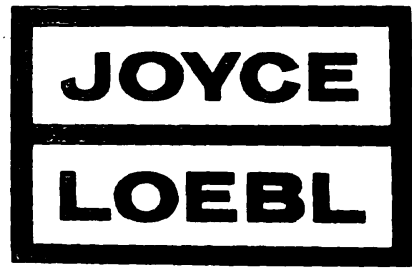

JOYCE-LOEBL

Gerätefechnik GmbH

7769 Kalkofen - Romhalden - Telefon 07557/380
Gastrokamera-

\section{Untersuchung}

\section{Grundlagen}

Untersuchungstechnik, Bildbeurteilung, Ergèbnisse

mit den Referaten des Ersten Gastrokamera-Seminars, 13.-15. Juni 1969, Berlin

\section{Herausgegeben}

\section{von Dozent Dr. H. Oshima}

Gastprofessor an der Freien Universität Berlin, Nippon-IkaUniversität Tokyo,

Präsident der European Ássociation for Gastrokamera Diagnosis, unter Mitarbeit von Dr. W. BergemanN, Berlin

Oktav. VIII, 146 Seiten. Mit 33 Abbildungen. 1971.

Kartoniert DM 20,-

Die Gastrokamera-Untersuchung, ein schnelles, einfaches und beschwerdearmes Verfahren zur intragastralen farbigen Fotografie für die Magen-Diagnostik hat in Europa in den letzten Jahren eine zunehmende Bedeutung erlangt.

Vom 13.-15. 6. 1969 wurde das Erste Gastrokamera-Seminar im Klinikum Steglitz der Freien Universität Berlin veranstaltet.

Die verschiedenen Gastrokamera-Modelle, die Untersuchungstechnik und besonders die Beurteilungsmethode sowie -kriterien wurden als Schwerpunkte behandelt.

Durch die Berichte der verschiedenen Untersucher aus 6 europäischen Ländern wurde der derzeitige Stand der GastrokameraDiagnostik in Europa dargelegt.

Die etwa 300 Teilnehmer aus 9 europäischen Ländern bewiesen das rege Interesse an dieser Untersuchungsart.

An einer weiter zunehmenden Bedeutung dieses diagnostischen Verfahrens ist bei der Häufigkeit von Magenerkrankungen, insbesondere des Magenkrebses nicht zu zweifeln. Dazu soll die Herausgabe dieser Verhandlungsberichte als Leitfaden der Gastrokamera-Untersuchung beitragen.

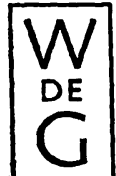




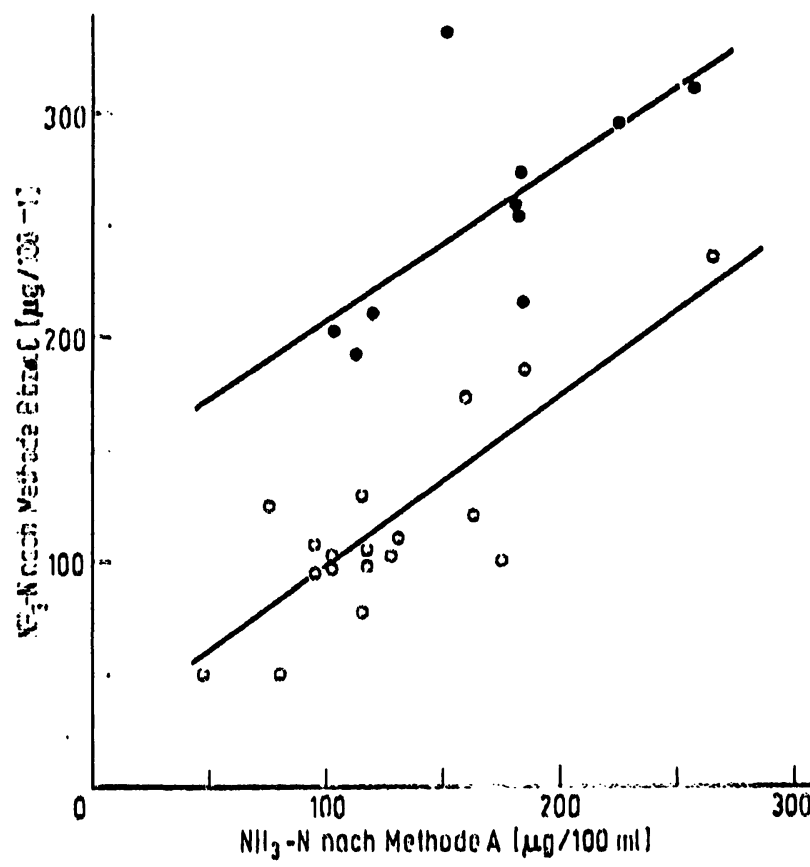

Al) 4

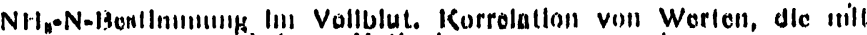

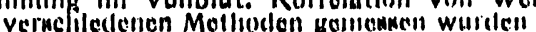

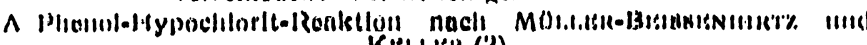
Kiti,1.1\&11 (2)

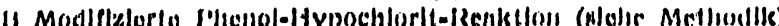

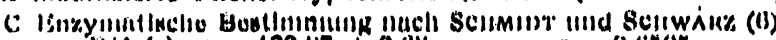

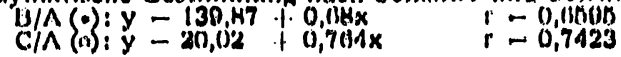

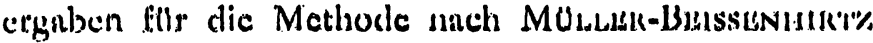
und Kwswe eluen Variationskoeffivienten von $1,83 \%$, fiir die Modifikntion cinen Wert von 2,01\% ("Tab. 3).

Dic Wisclerfindlungsesute von \#ugesctetem $\mathrm{NII}_{n}-\mathrm{N} \%$ Blut betrug fils die kolorimetrische Methesele (nach

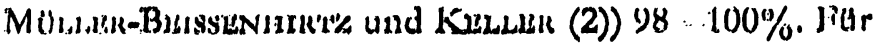
diesclbe Methode bestand eine streng lineare Beriehumy, "wwischen NI- $\mathrm{F}_{\mathrm{y}}-\mathrm{K}$ Konzentration der Stundadissung und

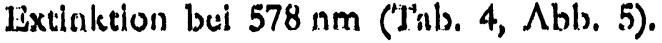

\section{Normumerto}

Die in der Literntur angegebenen Normwerte, die mit der: Milerodiffinsion nach Conway gewonnen wurden, liegen zwar in derselben Gxublenoxdnung, differieren aher erheblich in berag auf dic obere Normgrenze ('tals. 5). Es ist nicht sicher zu entscleciden, ol unterschiedliche Behnndlung der Blutprohen oder Gebrauch von Moslifikationen die Urwache ist. Pijpene Normwerte wurden unter Ausschalemng nller mëglichen lichler-

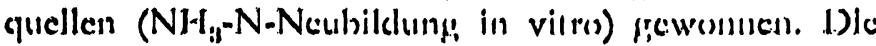
Blutentnahme erfolyte am nücliternen batienten bei

Tiuls. : I

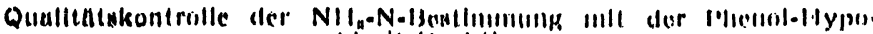

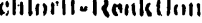

\begin{tabular}{|c|c|c|c|c|c|c|c|}
\hline \multirow[t]{2}{*}{ Probe Nr. } & \multirow[t]{2}{*}{$\|$} & \multicolumn{3}{|c|}{ 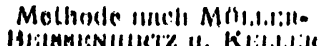 } & \multicolumn{3}{|c|}{ Mindlifalerio Mullende } \\
\hline & & & & - Kili,i.l & $x$ & H & V"ii \\
\hline \multicolumn{8}{|c|}{ 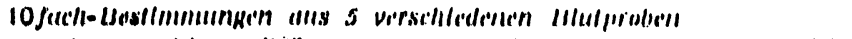 } \\
\hline $\begin{array}{l}1 \\
2 \\
11 \\
1 \\
1 \\
0\end{array}$ & $\begin{array}{l}10 \\
10 \\
10 \\
10 \\
10\end{array}$ & $\begin{array}{l}204 \\
1731 \\
1794 \\
21: 3 \\
1.11\end{array}$ & 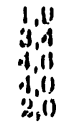 & 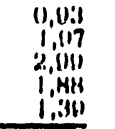 & 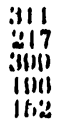 & $\begin{array}{l}4,7 \\
1,1 \\
1,2 \\
1,1 \\
1,7 \\
1,7\end{array}$ & 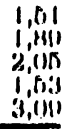 \\
\hline & & & & $81, \mathrm{~K} ; \mathrm{s}$ & & & 2,119 \\
\hline
\end{tabular}

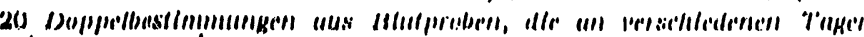

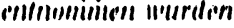

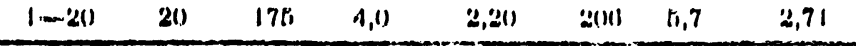

'I'al). I

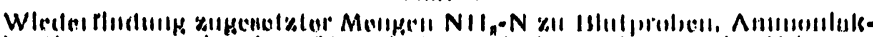

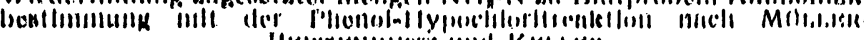

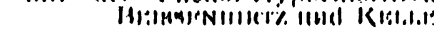

\begin{tabular}{|c|c|c|c|c|}
\hline \multirow{2}{*}{ 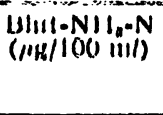 } & \multirow{2}{*}{ 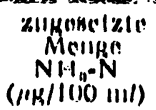 } & \multirow{2}{*}{ 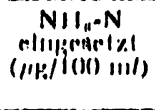 } & \multicolumn{2}{|c|}{ 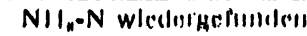 } \\
\hline & & & $(1 / k / 1000111 /)$ & $\left(n_{\prime \prime \prime)}\right)$ \\
\hline (11K & 100 & $111 \mathrm{H}$ & $2(1)$ & 101 \\
\hline IIN & $2(1)$ & 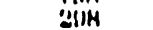 & נj(u) & 100,7 \\
\hline O4 & $3(0)$ & :I114 & :IIIIi & i(1)i, 2 \\
\hline SiN & 100) & AIIH & AIII) & $(N, 1$ \\
\hline IIN & (iv)(i) & IIIIH & IIIII) & UIH, K \\
\hline (1) & 800 & HINH & $H(1) 2$ & (101), :1 \\
\hline
\end{tabular}

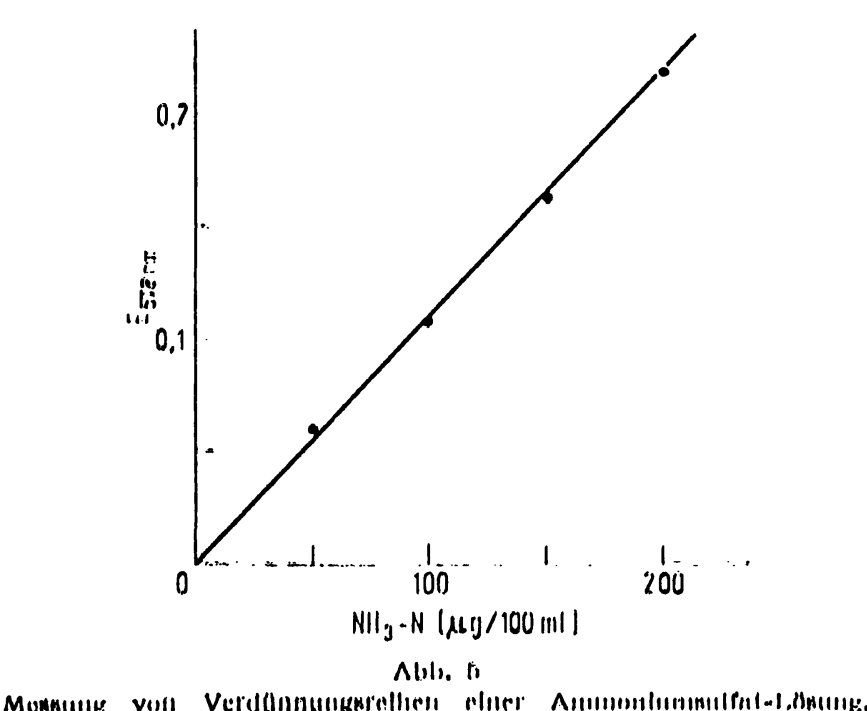

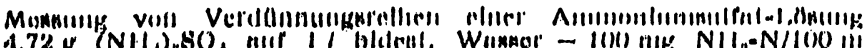

I'ill. $n$

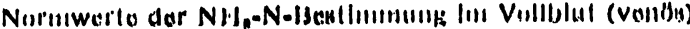

\begin{tabular}{|c|c|c|c|c|}
\hline Motlinto & 11 & 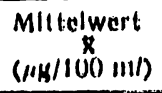 & 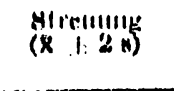 & Alilur \\
\hline 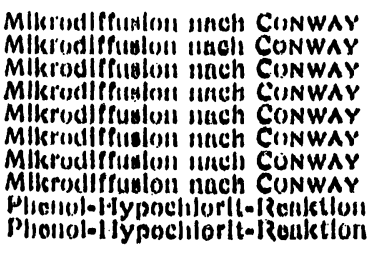 & $\begin{array}{l}100 \\
31 \\
122 \\
10 \\
10 \\
211 \\
10 \\
43 \\
24 \\
111 \\
11 \\
30\end{array}$ & 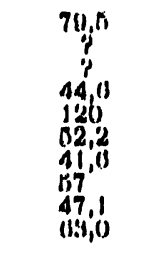 & 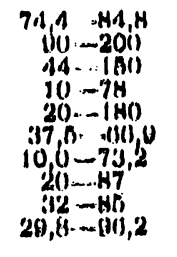 & \multirow[t]{2}{*}{ 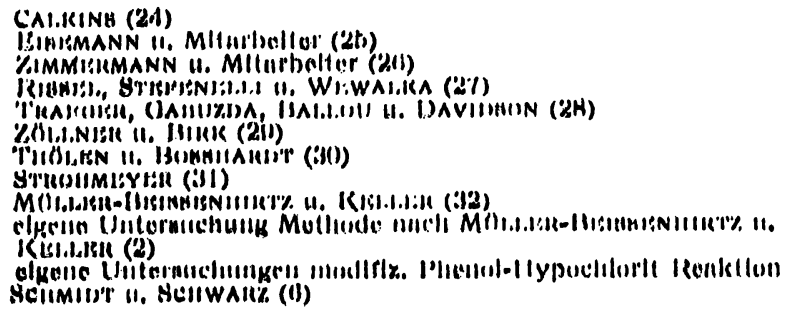 } \\
\hline 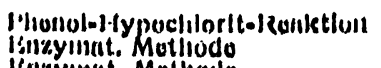 & $\prod_{p}^{10}$ & 07,0 & $\begin{array}{l}n(1,8)-13 H, 4 \\
J(1)=-40\end{array}$ & \\
\hline 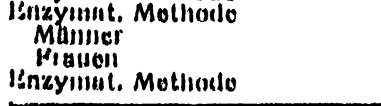 & $\begin{array}{l}120 \\
\text { HO } \\
18\end{array}$ & $\begin{array}{l}1122 \\
76) \\
\text { Hit, } 7\end{array}$ & $\begin{array}{c}2011-1111 \\
3010-1100 \\
22,0-100,0)\end{array}$ & 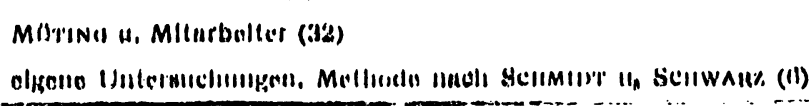 \\
\hline
\end{tabular}


völliger Körperruhe, nach mindestens 12 stdg. Nahrungskarenz. Die Werte, die mit der Methode nach MÜLLER-BEISSENHIRTZ und KeLlER gewonnen wurden, stimmen gut mit den Normwerten der Autoren überein. Sie sind bei exakter Einhaltung aller Versuchsbedingungen als reproduzierbare Normwerte, die den Messungen zugrundegelegt werden können, zu betrachten. Höhere Normwerte fanden wir in Übereinstimmung mit Müring und Mitarbeitern (Tab. 5) für die enzymatische Methode $\mathrm{NH}_{3}$-N-Neubildung während der Reaktion?) und für die Modifikation der PhenolHypochloritreaktion.

\section{Besprechung der Ergebnisse}

Wie die Untersuchungen von KELLER und Mitarbeitern $(2,10)$, sowie von Schmidt und Schwarz (6) gezeigt haben, können mit den direkten Verfahren der Ammoniakbestimmung im Blut reproduzierbare Werte gewonnen werden.

$\mathrm{Da}$ die aufwendige und'störanfällige Diffusion nach Conway nicht mehr exforderlich ist, sind die neuen Methoden einfacher zu handhaben und erlauben Untersuchungen an einem größeren Patientengut auch im Routinelaboratorium. Richtige Werte werden aber nur erhalten, wenn alle Fehlerquellen der Methoden beachtet werden. Die wesentlichsten Fehlermöglichkeiten betreffen die Teilschritte von der Probenentnahme bis zum Reaktionsansatz. Die Ammoniakneubildung in vitro, die sowohl im Nativblut als auch in enteiweißten Extrakten stattfindet, führt zu falsch hohen Werten. Sie läßt sich auch durch sofortige Neutralisation der sauren Extrakte nicht völlig verhindern. Es ist daher erforderlich, den Reaktionsansatz sofort im Anschlu $\beta$ an die Enteiweißung durchzuführen. Nach eigenen Erfahrungen beträgt die Zeitspanne von der Blutentnahme bis zum Ansatz bei optimalen Arbeitsbedingungen maximal 5-6 Min. Unter diesen Bedingungen beträgt der Fehler durch Ammoniakbildung in vitro etwa $2 \%$.
Eine Fehlerquelle der kolorimetrischen Methoden, die sich der Phenol-Hypochlorit-Reaktion bedienen, ist die Freisetzung von Ammoniak aus Aminosäuren $(3,18$, 20) und die Bildung von freiem Stickstoff aus Ammoniak durch das Hypochloritreagenz selbst (19). Der Umfang der Störung ist abhängig sowohl von der Struktur der Aminosäuren als auch von der Hypochloritkonzentration im Test (3). Unter den' gegebenen Testbedingungen spalten Aminosäuren nur einen sehr geringen Anteil ihres Stickstoffs ab, lediglich Glycin führt bereits in physiologischen Konzentrationen zu einer Verstärkung der Farbstoffbildung. Es muß aber auf jeden Fall darauf geachtet werden, daß der Gehalt der Hypochloritlösung an aktivem Chlor konstant gehalten wird und der Arbeitsvorschrift (10) genau entspricht.

Unterschiedliche Normwerte können durch Modifikation der Phenol-Hypochlorit-Methode bedingt sein. In eigenen Untersuchungen konnten wir zeigen, daß zwei Modifikationen dieser Methode zwar nur gering unterschiedliche Normwerte ergeben, $\mathrm{da} \beta$ aber die Korrelation der Werte besonders im oberen Bereich nicht optimal ist. Auch die Werte der enzymatischen Methode stimmen mit denen des kolorimetrischen Verfahrens im hohen Bereich nicht immer überein. Die enzymatische Methode, die frei von solchen Störungen ist, erfordert Übung und Kenntnisse enzymatischer Vorgänge, da der Endpunkt der Reaktion auch bei optimalem Einsatz an NADH und Glutamatdehydrogenase nicht immer einwandfrei zu erkennen ist.

Die Untersuchungen zeigen; $\mathrm{da} B$ auch die neuen Methoden der Blutammoniakbestimmung weiterer Verbesserung bedürfen. Eine wesentliche Verbesserung stellt die neuerdings von KELLER empfohlene Mikrobestimmung im Kapillarblut dar, die auch die Fehlerquellen der Ammoniakneubildung berücksichtigt (10). $\mathrm{Ob}$ die mit dieser Methode gewonnenen Werte dem klinischen Bild besser entsprechen als diejenigen des venösen Bluts, wäre zu überprüfen.

\section{Literatur}

1. Conway, E. J., Biochem. J. 29, 2755 (1935). - 2. MüllerBeissenhirtz, W. und H. Keller, Klin. Wschr. 43, 43 (1965). 3. Fürth, O., A. FrIedrich und R. Scholl, Biochem. Z. 240, 50 (1931). - 4. Breuer, H., Persönliche Mitteilung. - 5. Kirsten, E., C. Gerez und R. Kirsten, Biochem. Z. 337, 312 (1963). 6. Schmidt, F. H. und M. Schwarz, Klin. Wschr., 591,44 (1966). 7. Müller-BeissenhiRtz, W., Z. analyt. Chem. 212, 145 (1965). 8. Chaney, A. L. und E. P. Marbach, Clin. Chem. (New York), 8, 130 (1962). - 9. Searcy, R. L., G. S. Gough, J. L. Krotzer und L. M. Bergquist, Amer. J. Med. Technol. 27, 255 (1961). 10. Keller, H., W. Müller-Beissenhirtz und E. Neumann, Klin. Wschr. 45, 314 (1967). - 11. Conway, E. J. und R. Cooke, Biochem. J. 33, 457 (1939). - 12. Conway, E. J. und R. Cooke, Nature (London), 142, 720 (1938). - 13. Conwax, E. J., Microdiffusion. Analysis and volumetric error. 3rd Edition, Crosby, Lockwood and Son, London (1950). - 14. Conway, E. J., Micodiffusion. Analysis and volumetric error 4th, revised ed., Crosby, Lockwood 81 td. London, (1957). - 15. SeEgmiller, J. E., R. Schwartz und C. S. Davidson, J. clin. Invest. 33, 984 954). -(1 16. Krebs, H. A., L. V. Egglestone und R. HeMrs,
Biochem. J. 44, 159 (1949), zit. nach Brown R. H., Duda G. D., Korkes, S. und Ph. Handler, Atch. Biochem. Biophysics 66 , 301 (1957). - 17. ENGEL, R., zit. nach (3). - 18. LANGHELD, R., zit. nach (3). - 19. Raschig, zit. nach (3). - 20. Forman, D. T., Clin. Chem. (New York) 10, 496, (1964). - 21. Seligson, D. und Hirahara, K., J. Laborat. Clin. Med. S. Louis, 49, 962 (1957). 22. Mriler, G. E. und J. D. Rice, Amer. J. Clin. Path. 39, 97 (1963). - 23. KIRK, W., Acta med. scand. Suppl. 77, 1, (1936). 24. Calkins, A., J. Laborat. Clin. Med. S. Louis, 47, 343 (1956). 25. Eiseman, B., W. Bakewell und G. Clark, Amer. J. Med. 20, 87 (1956). - 26. ZimmermanN, H. J. und R. J. Korn, Amer. J. Med. Sci. 231, 177 (1956). - 27. RisSEL, STEFENELLI und Wewalka, Wien. Klin. Wschr. 69, 172 (1957). - 28. Traeger, Gabuzda, Ballou und Davidson, Metabolism 3, 99 (1954). 29. ZöllNer, N. und H, BrRK, Klin. Wschr. 36, 12 (1958). -30. Thölen H. und R. Bosshard, Klin. Wschr. 36, 574 (1958). 31. StrohmeYer, G., Verhandl. internat. Kongreß $f$. Innere Medizin, München 1962, Verhandlungsband Stuttgart (1963). 32. Müring, D., J. Heinze, J. Reikowski und G. Betzien, Clin. Chim. Acta Amsterdam, 19, 391 (1968).

Frau Prof. Dr. A. Englhardt, 355 Marburg/Lahn, Mannkopffstr. 1 